\title{
Methylenetetrahydrofolate reductase tagging polymorphisms are associated with risk of non-small cell lung cancer in eastern Chinese Han population
}

\author{
Hao Ding ${ }^{1, *}$, Yafeng Wang ${ }^{2, *}$, Yuanmei Chen $^{3, *}$, Chao Liu ${ }^{4}$, Hao Qiu ${ }^{5}$, Mingqiang \\ Kang ${ }^{6}$ and Weifeng Tang ${ }^{6}$ \\ ${ }^{1}$ Department of Respiratory Disease, Affiliated People's Hospital of Jiangsu University, Zhenjiang, Jiangsu Province, China \\ ${ }^{2}$ Department of Cardiology, The People's Hospital of Xishuangbanna Dai Autonomous Prefecture, Jinghong, Yunnan Province, \\ China \\ ${ }^{3}$ Department of Thoracic Surgery, Fujian Cancer Hospital, Fujian Medical University Cancer Hospital, Fuzhou, Fujian Province, \\ China \\ ${ }^{4}$ Department of Cardiothoracic Surgery, Affiliated People's Hospital of Jiangsu University, Zhenjiang, Jiangsu Province, China \\ ${ }^{5}$ Department of Immunology, School of Medicine, Jiangsu University, Zhenjiang, Jiangsu Province, China \\ ${ }^{6}$ Department of Thoracic Surgery, Fujian Medical University Union Hospital, Fuzhou, Fujian Province, China \\ *These authors contributed equally to this work \\ Correspondence to: Weifeng Tang, email: twf001001@126.com \\ Mingqiang Kang, email: Mingqiang_Kang@126.com
}

Keywords: MTHFR; polymorphism; non-small cell lung cancer; risk

Received: May 30, 2017 Accepted: November 08, 2017 Published: December 04, 2017

Copyright: Ding et al. This is an open-access article distributed under the terms of the Creative Commons Attribution License 3.0 (CC BY 3.0), which permits unrestricted use, distribution, and reproduction in any medium, provided the original author and source are credited.

\section{ABSTRACT}

Previous reports implicated 5,10-ethylenetetrahydrofolate reductase (MTHFR) polymorphisms acted as a potential risk factor for several cancers. In order to explore the effect of MTHFR SNPs on non-small cell lung cancer (NSCLC), we selected MTHFR tagging single nucleotide polymorphisms (SNPs) and carried out a case-control study to determine the potential relationship of MTHFR SNPs with NSCLC risk. Our study consisted of 521 NSCLC patients and 1,030 non-cancer controls. MTHFR SNPs were genotyped by SNPscan ${ }^{\mathrm{TM}}$ genotyping assay. Using four genetic models (additive, Homozygote, dominant, recessive), the genotype frequencies were compared using the chi-squared $\left(X^{2}\right)$ test. Crude/adjusted odds ratios (ORs) with their $95 \%$ confidence intervals (CIs) were used to assess the difference for the genotype distribution. We found that MTHFR rs1801133 G>A polymorphism decreased the risk of overall NSCLC. In a subgroup analysis, MTHFR rs $1801133 \mathrm{G}>\mathrm{A}$ polymorphism also decreased NSCLC risk in female, $<60$ years and never smoking subgroups. However, we identified that MTHFR rs4845882 G>A polymorphism was associated with the development of NSCLC in female subgroup. In addition, MTHFR rs9651118 T>C polymorphism increased the risk of NSCLC in $<60$ years, never smoking and BMI $<24 \mathrm{~kg} / \mathrm{m}^{2}$ subgroups. In conclusion, the current study highlights MTHFR rs $1801133 \mathrm{G}>\mathrm{A}$ variants decreases the risk of NSCLC. Nevertheless, MTHFR rs4845882 G>A and rs9651118 $\mathrm{T}>\mathrm{C}$ polymorphisms may be associated with NSCLC susceptibility. Well-designed large-scale studies are needed to confirm these findings and explore the interactions of gene-gene and gene-environment involved in MTHFR SNPS and NSCLC.

\section{INTRODUCTION}

Lung cancer (LC) caused by multiple risk factors is one of the common malignancies worldwide. With very complex biological characteristics and high degree of invasiveness, it is difficult to diagnose at an early stage and lack of very effective treatment at an advanced stage. Thus, LC is a common public health problem with a poor 
prognosis. LC involves two major subtypes, such as small cell LC and non-small cell LC (NSCLC). In addition, NSCLC cases account for most of the total LC cases. The increasing incidence of NSCLC is closely related to tobacco consumption, air pollution, cooking fumes, asbestos and other environmental factors [1]. However, these known risk factors might not contribute to overall susceptibility to NSCLC. Recently, individual's genetic factors have also been determined to cause NSCLC.

Folic acid, or 5-methyltetrahydrofolate, is a cofactor in the metabolism of homocysteine to methionine [2]. 5,10-ethylenetetrahydrofolate reductase (MTHFR) catalyzed reduction of 5,10-methylenetetrahydrofolate (methylene-THF), a donor of methyl for dUMP to dTMP transform, to methyl-THF, the primary methyl donor in methionine synthesis. Methionine is transformed to S-adenosyl-1-methionine (SAM) which is the principal methyl donor in over 100 biochemical responses, including cytosine methylation in DNA. By the catalysis of DNA (cytosine-5)-methyltransferase, methyl group of SAM was transferred to $\mathrm{C} 5$ of cytosine within $\mathrm{CpG}$ island in the genomic DNA in higher eukaryotes [3-5]. MTHFR is a dimeric flavoprotein in human and each monomer is bound to flavinadenosine-dinucleotide noncovalently [6]. Each monomer contains a N-terminal catalytic domain that binds the allosteric SAM inhibitory regulating enzyme activity in response to the methionine levels in the cell [7].The findings of the relationship between methylation patterns and folate status in individuals with cancer and healthy normal individuals provide stronger evidences for a mechanism by which folate may modify DNA methylation and alter the risk of cancer.

Methylenetetrahydrofolate reductase (MTHFR), whose gene maps to the short arm of Chromosome 1 and encodes a 77-kDa protein with 656 amino acids. Many single nucleotide polymorphisms (SNPs) have been identified (http://www.ncbi.nlm.nih.gov/SNP), such as rs1801131, rs1801133, rs1537514, rs9651118, rs1537516, rs3753584, rs4845882, rs4846048, rs2066462 and rs3737967 polymorphisms, etc. A number of case-control studies focused on the association between MTHFR polymorphisms and the risk of LC [8$15]$, however, the results were inconsistent. For example, a meta-analysis suggested that MTHFR rs1801133 G>A was not associated the risk of LC in Chinese population [16]. Nevertheless, Yang et al. reported that MTHFR rs1801133 $\mathrm{G}>\mathrm{A}$ polymorphism increased the risk of lung cancer in Asians, but not in Caucasians [17]. These ambiguous findings may be due to the limited sample size or difference in populations. In order to extensively explore the relationship of MTHFR SNPs with LC susceptibility, we selected MTHFR tagging SNPs $(\mathrm{rs} 3753584 \mathrm{~T}>\mathrm{C}, \quad r s 4845882 \mathrm{G}>\mathrm{A}, \quad \mathrm{rs} 1801133 \mathrm{G}>\mathrm{A}$, rs4846048 $\mathrm{A}>\mathrm{G}$ and $\mathrm{rs} 9651118 \mathrm{~T}>\mathrm{C}$ ) and carried out a case-control study to determine the potential effect of MTHFR SNPs on NSCLC risk.

\section{RESULTS}

\section{Baseline characteristics}

In this study, a total of 521 sporadic NSCLC patients and 1,030 normal controls were enrolled. Age and sex were full matched $(P=0.843$ and $P=0.453$, respectively; Table 1). Of the NSCLC patients, 287 were male and 234 were female, with a mean age of $59.76 \pm 10.71$ years. The non-cancer controls were comprised of 588 males and 442 females with a mean age of $60.34 \pm 9.11$ years. Of the tobacco consumption and drinking and body mass index (BMI), differences were found between NSCLC patients and non-cancer controls $(P<0.001$, Table 1$)$. The genotype distribution of MTHFR was calculated after genotyping the 1,551 included participants. For MTHFR rs 1801133 $\mathrm{G}>\mathrm{A}, \mathrm{rs} 4845882 \mathrm{G}>\mathrm{A}, \mathrm{rs} 4846048 \mathrm{~A}>\mathrm{G}, \mathrm{rs} 3753584 \mathrm{~T}>\mathrm{C}$ and rs9651118 $\mathrm{T}>\mathrm{C}$ polymorphisms, success rates of genotyping were $99.87 \%, 99.94 \%, 99.94 \%, 99.94 \%$ and $99.94 \%$, respectively (Table 2). The genotype distribution of MTHFR SNPs reached Hardy-Weinberg equilibrium (HWE) in controls, except for MTHFR rs4846048 A>G polymorphism $(P=0.036)$ (Table 2$)$.

\section{Association of MTHFR rs1801133 G>A, rs4845882 $G>A, r s 4846048 A>G, r s 3753584 T>C$ and rs9651118 $\mathrm{T}>\mathrm{C}$ polymorphisms with the development of NSCLC}

Table 3 summarizes the genotypes of MTHFR SNPs. MTHFR rs1801133 G>A polymorphism decreased the risk of NSCLC in two genetic models [AA vs. GG: crude odds ratio $(\mathrm{OR})=0.66,95 \%$ confidence interval (CI): $0.45-0.96, P=0.031$; and AA vs. GA/GG: crude $\mathrm{OR}=0.69,95 \% \mathrm{CI}: 0.48-0.99, P=0.042$; Table 3$]$. Adjustment for age, sex, BMI, smoking and drinking, the decreased risk of NSCLC was also found (AA vs. GG: adjusted $\mathrm{OR}=0.66,95 \% \mathrm{CI}$ : $0.47-0.97, P=0.035$; Table $3)$. However, the above findings were not significant after the Bonferroni correction for multiple comparisons. For MTHFR rs3753584 T $>$ C, rs4845882 $\mathrm{G}>\mathrm{A}, \mathrm{rs} 4846048$ $\mathrm{A}>\mathrm{G}$ and $\mathrm{rs} 9651118 \mathrm{~T}>\mathrm{C}$ polymorphisms, we found null association between these SNPs and the risk of NSCLC (Table 3).

\section{Association of $M T H F R$ rs1801133 G>A, rs4845882 $\mathrm{G}>\mathrm{A}$, rs4846048 $\mathrm{A}>\mathrm{G}$, rs3753584 $\mathrm{T}>\mathrm{C}$ and rs9651118 $\mathrm{T}>\mathrm{C}$ polymorphisms with the development of NSCLC in Different Stratification Groups}

After adjustment by logistic regression analysis, we found MTHFR rs1801133 G>A variants were associated with the decreased risk of NSCLC in some subgroups (female group: AA vs. GG: adjusted OR $=0.53,95 \% \mathrm{CI}$ $0.30-0.94, P=0.031$ and $\mathrm{AA} v$ s. GA/GG: adjusted $\mathrm{OR}=$ 
Table 1: Distribution of selected demographic variables and risk factors in NSCLC cases and controls

\begin{tabular}{|c|c|c|c|}
\hline \multirow{2}{*}{ Variable } & Overall Cases $(n=521)$ & Overall Controls $(n=1,030)$ & \multirow{2}{*}{$P^{a}$} \\
\hline & $n(\%)$ & $n(\%)$ & \\
\hline Age (years) & $59.76 \pm 10.71$ & $60.34 \pm 9.11$ & 0.268 \\
\hline Age (years) & & & 0.843 \\
\hline$<60$ & $238(45.68)$ & $476(46.21)$ & \\
\hline$\geq 60$ & $283(54.32)$ & $554(53.79)$ & \\
\hline Sex & & & 0.453 \\
\hline Male & $287(55.09)$ & $588(57.09)$ & \\
\hline Female & $234(44.91)$ & $442(42.91)$ & \\
\hline Smoking status & & & $<0.001$ \\
\hline Never & $317(60.84)$ & $828(80.39)$ & \\
\hline Ever & $204(39.16)$ & $202(19.61)$ & \\
\hline Alcohol use & & & $<0.001$ \\
\hline Never & $444(85.22)$ & $949(92.14)$ & \\
\hline Ever & 77 (14.78) & $81(7.86)$ & \\
\hline BMI $\left(\mathrm{kg} / \mathrm{m}^{2}\right)$ & $23.00( \pm 3.03)$ & $23.84( \pm 3.06)$ & $<0.001$ \\
\hline \multicolumn{4}{|l|}{ BMI $\left(\mathrm{kg} / \mathrm{m}^{2}\right)$} \\
\hline$<24$ & 337 (64.68) & $547(53.11)$ & $<0.001$ \\
\hline$\geq 24$ & $184(35.32)$ & $483(46.89)$ & \\
\hline
\end{tabular}

${ }^{a}$ Two-sided $\chi^{2}$ test and Student $t$ test

Table 2: Primary information for MTHFR polymorphisms (rs1801133 G>A, rs9651118 T $>$ C rs4845882 $\mathrm{G}>\mathrm{A}$, rs4846048 $\mathrm{A}>\mathrm{G}$ and $\mathbf{r s 3 7 5 3 5 8 4} \mathrm{T}>\mathrm{C}$ )

\begin{tabular}{lccccc}
\hline \multicolumn{1}{c}{ Genotyped SNPs } & $\begin{array}{c}\text { rs1801133 } \\
\mathbf{G}>\mathbf{A}\end{array}$ & $\begin{array}{c}\mathbf{r s 3 7 5 3 5 8 4} \\
\mathbf{T}>\mathbf{C}\end{array}$ & $\begin{array}{c}\mathbf{r s 4 8 4 5 8 8 2} \\
\mathbf{G}>\mathbf{A}\end{array}$ & $\begin{array}{c}\mathbf{r s 4 8 4 6 0 4 8} \\
\mathbf{A}>\mathbf{G}\end{array}$ & $\begin{array}{c}\text { rs9651118 } \\
\mathbf{T}>\mathbf{C}\end{array}$ \\
\hline Chromosome & 1 & 1 & 1 & 1 & 1 \\
Function & Missense & NearGene-5 & Intron & Intron & Intron \\
Chr Pos (Genome Build 36.3) & 11778965 & 11787173 & 11765754 & 11768839 & 11784801 \\
MAF for Chinese in database $_{\text {MAF in our controls }(\boldsymbol{n}=1,030)}$ & 0.439 & 0.093 & 0.198 & 0.105 & 0.382 \\
$P_{\text {value for HWE test in our controls }}$ & 0.345 & 0.118 & 0.214 & 0.095 & 0.383 \\
Genotyping method & 0.947 & 0.712 & 0.454 & 0.036 & 0.081 \\
\% Genotyping value & SNPscan & SNPscan & SNPscan & SNPscan & SNPscan \\
\hline
\end{tabular}

${ }^{\mathrm{a}} \mathrm{MAF}:$ minor allele frequency;

${ }^{b} H W E$ : Hardy-Weinberg equilibrium

$0.58,95 \%$ CI $0.33-1.00, P=0.048 ;<60$ years subgroup: AA vs. GG: adjusted OR $=0.53,95 \%$ CI $0.28-1.00, P=$ 0.048 ; never smoking group: AA vs. GG: adjusted $\mathrm{OR}=$ $0.58,95 \%$ CI $0.36-0.93, P=0.024$ and AA vs. GA/GG: adjusted $\mathrm{OR}=0.62,95 \% \mathrm{CI} 0.39-0.99, P=0.044$; Table 4 ).

The correlation between MTHFR rs $3753584 \mathrm{~T}>\mathrm{C}$ polymorphism and NSCLC risk in the stratified analyses are summarized Table 5. We found that MTHFR rs 3753584 $\mathrm{T}>\mathrm{C}$ vatiants were not associated with the susceptibility of NSCLC in any subgroup (Table 5).

The relationship of MTHFR rs4845882 G>A polymorphism with NSCLC susceptibility in the stratified analysis is listed in Table 6. We identified that MTHFR rs4845882 G>A polymorphism was associated with the development of NSCLC in female subgroup (GA vs. GG: adjusted $\mathrm{OR}=1.47,95 \%$ CI $1.05-2.05, P=0.025$ ).

Table 7 demonstrated that MTHFR rs $4846048 \mathrm{~A}>\mathrm{G}$ polymorphism was not associated with the development of NSCLC in any subgroup.

We found that MTHFR rs9651118 $\mathrm{T}>\mathrm{C}$ polymorphism increased the risk of NSCLC in several stratified analyses ( $<60$ years group: CC $v s$. TT: adjusted $\mathrm{OR}=1.64,95 \% \mathrm{CI} 1.00-2.69, P=0.049$ and $\mathrm{CC} v s . \mathrm{TC} /$ TT: adjusted $\mathrm{OR}=1.75,95 \% \mathrm{CI} 1.12-2.74, P=0.014$; 
Table 3: Logistic regression analyses of associations between $M T H F R$ rs1801133 $\mathbf{G}>\mathbf{A}$, rs3753584 $\mathrm{T}>\mathrm{C}$, rs4845882 $\mathrm{G}>\mathrm{A}, \mathrm{rs4846048} \mathrm{A}>\mathrm{G}$ and $\mathrm{rs9651118} \mathrm{T}>\mathrm{C}$ polymorphisms and the risk of NSCLC

\begin{tabular}{|c|c|c|c|c|c|c|c|c|}
\hline \multirow[t]{2}{*}{ Genotype } & \multicolumn{2}{|c|}{$\begin{array}{c}\text { Cases } \\
(n=521)\end{array}$} & \multicolumn{2}{|c|}{$\begin{array}{c}\text { Controls } \\
(n=1,030)\end{array}$} & \multirow[t]{2}{*}{$\begin{array}{c}\text { Crude OR } \\
(95 \% \mathrm{CI})\end{array}$} & \multirow[t]{2}{*}{$\boldsymbol{P}$} & \multirow[t]{2}{*}{$\begin{array}{c}\text { Adjusted OR a } \\
(95 \% \mathrm{CI})\end{array}$} & \multirow[t]{2}{*}{$P$} \\
\hline & $n$ & $\%$ & $n$ & $\%$ & & & & \\
\hline \multicolumn{9}{|c|}{ MTHFR rs1801133 G>A } \\
\hline GG & 241 & 46.35 & 441 & 42.86 & 1.00 & & 1.00 & \\
\hline GA & 235 & 45.19 & 466 & 45.29 & $0.92(0.74-1.15)$ & 0.467 & $0.92(0.73-1.16)$ & 0.461 \\
\hline $\mathrm{AA}$ & 44 & 8.46 & 122 & 11.86 & $0.66(0.45-0.96)$ & 0.031 & $0.66(0.44-0.97)$ & 0.035 \\
\hline $\mathrm{GA}+\mathrm{AA}$ & 279 & 53.65 & 588 & 57.14 & $0.87(0.70-1.07)$ & 0.192 & $0.87(0.70-1.08)$ & 0.207 \\
\hline $\mathrm{GG}+\mathrm{GA}$ & 476 & 91.54 & 907 & 88.14 & 1.00 & & 1.00 & \\
\hline $\mathrm{AA}$ & 44 & 8.46 & 122 & 11.86 & $0.69(0.48-0.99)$ & 0.042 & $0.69(0.47-1.00)$ & 0.050 \\
\hline A allele & 323 & 31.06 & 710 & 34.50 & & & & \\
\hline \multicolumn{9}{|c|}{ MTHFR rs3753584 T>C } \\
\hline TT & 403 & 77.35 & 800 & 77.75 & 1.00 & & 1.00 & \\
\hline $\mathrm{CT}$ & 111 & 21.31 & 216 & 20.99 & $1.02(0.79-1.32)$ & 0.872 & $1.03(0.79-1.35)$ & 0.829 \\
\hline $\mathrm{CC}$ & 7 & 1.34 & 13 & 1.26 & $1.07(0.42-2.71)$ & 0.885 & $1.04(0.39-2.76)$ & 0.937 \\
\hline $\mathrm{CT}+\mathrm{CC}$ & 118 & 22.65 & 229 & 22.25 & $1.02(0.80-1.32)$ & 0.860 & $1.03(0.79-1.34)$ & 0.826 \\
\hline $\mathrm{TT}+\mathrm{CT}$ & 514 & 98.66 & 1,016 & 98.74 & 1.00 & & 1.00 & \\
\hline $\mathrm{CC}$ & 7 & 1.34 & 13 & 1.26 & $1.07(0.42-2.69)$ & 0.894 & $1.03(0.39-2.74)$ & 0.948 \\
\hline $\mathrm{C}$ allele & 125 & 12.00 & 242 & 11.76 & & & & \\
\hline \multicolumn{9}{|c|}{$M T H F R$ rs4845882 G>A } \\
\hline GG & 309 & 59.31 & 632 & 61.42 & 1.00 & & 1.00 & \\
\hline GA & 191 & 36.66 & 354 & 34.40 & $1.11(0.89-1.38)$ & 0.378 & $1.12(0.89-1.42)$ & 0.326 \\
\hline AA & 21 & 4.03 & 43 & 4.18 & $1.00(0.58-1.72$ & 0.999 & $1.14(0.65-2.01)$ & 0.642 \\
\hline $\mathrm{GA}+\mathrm{AA}$ & 212 & 40.69 & 397 & 38.58 & $1.09(0.88-1.35)$ & 0.422 & $1.12(0.90-1.10)$ & 0.308 \\
\hline $\mathrm{GG}+\mathrm{GA}$ & 500 & 95.97 & 986 & 95.82 & 1.00 & & 1.00 & \\
\hline $\mathrm{AA}$ & 21 & 4.03 & 43 & 4.18 & $0.96(0.57-1.64)$ & 0.891 & $1.09(0.63-1.91)$ & 0.753 \\
\hline A allele & 233 & 22.36 & 440 & 21.38 & & & & \\
\hline \multicolumn{9}{|c|}{$M T H F R$ rs4846048 A>G } \\
\hline AA & 428 & 82.15 & 849 & 82.51 & 1.00 & & 1.00 & \\
\hline $\mathrm{AG}$ & 90 & 17.27 & 165 & 16.03 & $1.08(0.82-1.44)$ & 0.578 & $1.13(0.84-1.51)$ & 0.423 \\
\hline GG & 3 & 0.58 & 15 & 1.46 & $0.40(0.11-1.38)$ & 0.146 & $0.48(0.13-1.73)$ & 0.264 \\
\hline $\mathrm{AG}+\mathrm{GG}$ & 93 & 17.85 & 180 & 17.49 & $1.03(0.78-1.35)$ & 0.861 & $1.08(0.81-1.44)$ & 0.609 \\
\hline $\mathrm{AA}+\mathrm{AG}$ & 518 & 99.42 & 1,014 & 98.54 & 1.00 & & 1.00 & \\
\hline GG & 3 & 0.58 & 15 & 1.46 & $0.39(0.11-1.36)$ & 0.140 & $0.47(0.13-1.70)$ & 0.250 \\
\hline $\mathrm{G}$ allele & 96 & 9.21 & 195 & 9.48 & & & & \\
\hline \multicolumn{9}{|c|}{ MTHFR rs9651118 T $>C$} \\
\hline $\mathrm{TT}$ & 187 & 35.89 & 378 & 36.73 & 1.00 & & 1.00 & \\
\hline $\mathrm{TC}$ & 245 & 47.02 & 513 & 49.85 & $0.97(0.77-1.22)$ & 0.783 & $0.94(0.74-1.20)$ & 0.636 \\
\hline $\mathrm{CC}$ & 89 & 17.08 & 138 & 13.41 & $1.31(0.95-1.80)$ & 0.100 & $1.30(0.93-1.81)$ & 0.124 \\
\hline $\mathrm{TC}+\mathrm{CC}$ & 334 & 64.11 & 651 & 63.27 & $1.04(0.83-1.29)$ & 0.745 & $1.02(0.81-1.28)$ & 0.895 \\
\hline $\mathrm{TT}+\mathrm{TC}$ & 432 & 82.92 & 891 & 86.59 & 1.00 & & 1.00 & \\
\hline $\mathrm{CC}$ & 89 & 17.08 & 138 & 13.41 & $1.33(1.00-1.78)$ & 0.054 & $1.34(0.99-1.82)$ & 0.057 \\
\hline $\mathrm{C}$ allele & 423 & 40.60 & 789 & 38.34 & & & & \\
\hline
\end{tabular}


Table 4: Stratified analyses between $M T H F R$ rs1801133 G>A polymorphism and NSCLC risk by sex, age, BMI, smoking status and alcohol consumption

\begin{tabular}{|c|c|c|c|c|c|c|c|c|}
\hline \multirow[t]{2}{*}{ Variable } & \multicolumn{3}{|c|}{$\begin{array}{c}\text { MTHFR rs1801133 G }>\text { A (case/ } \\
\text { control) }^{\mathrm{a}}\end{array}$} & \multicolumn{5}{|c|}{ Adjusted $\mathrm{OR}^{\mathrm{b}}(95 \% \mathrm{CI}) ; P$} \\
\hline & GG & GA & $\mathbf{A A}$ & GG & GA & $\mathbf{A A}$ & GA/AA & AA vs. (GA/GG) \\
\hline \multicolumn{9}{|l|}{ Sex } \\
\hline Male & $125 / 254$ & $136 / 275$ & $25 / 58$ & 1.00 & $\begin{array}{c}0.99(0.72-1.35) ; \\
P: 0.930\end{array}$ & $\begin{array}{c}0.83(0.48-1.45) ; \\
P: 0.518\end{array}$ & $\begin{array}{l}0.97(0.71-1.32) \\
\quad P: 0.840\end{array}$ & $\begin{array}{c}0.84(0.50-1.43) \\
P: 0.527\end{array}$ \\
\hline Female & $116 / 187$ & $99 / 191$ & $19 / 64$ & 1.00 & $\begin{array}{l}0.85(0.61-1.20) \\
\quad P: 0.357\end{array}$ & $\begin{array}{c}0.53(0.30-0.94) \\
\text { P: } 0.031\end{array}$ & $\begin{array}{l}0.78(0.56-1.07) \\
\quad P: 0.126\end{array}$ & $\begin{array}{c}0.58(0.33-1.00) \\
\text { P: } 0.048\end{array}$ \\
\hline \multicolumn{9}{|l|}{ Age } \\
\hline$<60$ & $125 / 213$ & $97 / 213$ & $16 / 49$ & 1.00 & $\begin{array}{c}0.79(0.56-1.12) \\
P: 0.187\end{array}$ & $\begin{array}{l}0.53(0.28-1.00) \\
\text { P: } 0.048\end{array}$ & $\begin{array}{l}0.74(0.53-1.03) \\
\quad P: 0.072\end{array}$ & $\begin{array}{c}0.59(0.32-1.09) \\
P: 0.090\end{array}$ \\
\hline$\geq 60$ & $116 / 228$ & $138 / 253$ & $28 / 73$ & 1.00 & $\begin{array}{c}1.05(0.77-1.44) \\
P: 0.759\end{array}$ & $\begin{array}{c}0.78(0.47-1.30) \\
P: 0.344\end{array}$ & $\begin{array}{c}1.01(0.74-1.36) \\
P: 0.976\end{array}$ & $\begin{array}{l}0.77(0.47-1.24) \\
\quad P: 0.276\end{array}$ \\
\hline \multicolumn{9}{|c|}{ Smoking status } \\
\hline Never & $153 / 352$ & $137 / 372$ & $26 / 103$ & 1.00 & $\begin{array}{l}0.86(0.65-1.14) \\
\quad P: 0.298\end{array}$ & $\begin{array}{l}0.58(0.36-0.93) \\
\quad \text { P: } 0.024\end{array}$ & $\begin{array}{l}0.81(0.62-1.05) \\
\quad P: 0.112\end{array}$ & $\begin{array}{l}0.62(0.39-0.99) \\
\quad \text { P: } 0.044\end{array}$ \\
\hline Ever & $88 / 89$ & 98/94 & $18 / 19$ & 1.00 & $\begin{array}{c}1.06(0.70-1.60) ; \\
P: 0.786\end{array}$ & $\begin{array}{c}0.92(0.45-1.90) \\
P: 0.829\end{array}$ & $\begin{array}{c}1.04(0.70-1.54) \\
P: 0.861\end{array}$ & $\begin{array}{c}0.90(0.45-1.78) \\
P: 0.754\end{array}$ \\
\hline \multicolumn{9}{|l|}{$\begin{array}{l}\text { Alcohol } \\
\text { consumption }\end{array}$} \\
\hline Never & $210 / 411$ & $197 / 426$ & $36 / 111$ & 1.00 & $\begin{array}{c}0.93(0.72-1.19) \\
P: 0.543\end{array}$ & $\begin{array}{c}0.68(0.44-1.03) ; \\
P: 0.071\end{array}$ & $\begin{array}{c}0.88(0.70-1.12) \\
\quad P: 0.293\end{array}$ & $\begin{array}{c}0.71(0.47-1.06) \\
P: 0.092\end{array}$ \\
\hline Ever & $31 / 30$ & $38 / 40$ & $8 / 11$ & 1.00 & $\begin{array}{c}0.88(0.44-1.75) \\
P: 0.711\end{array}$ & $\begin{array}{c}0.60(0.21-1.75) \\
P: 0.353\end{array}$ & $\begin{array}{l}0.82(0.42-1.58) \\
\quad P: 0.550\end{array}$ & $\begin{array}{c}0.65(0.24-1.75) \\
P: 0.393\end{array}$ \\
\hline \multicolumn{9}{|l|}{$\operatorname{BMI}\left(\mathrm{kg} / \mathrm{m}^{2}\right)$} \\
\hline$<24$ & $159 / 242$ & $151 / 247$ & $27 / 57$ & 1.00 & $\begin{array}{l}0.94(0.70-1.27) \\
\quad P: 0.699\end{array}$ & $\begin{array}{c}0.68(0.41-1.15) ; \\
P: 0.149\end{array}$ & $\begin{array}{c}0.89(0.67-1.19) \\
P: 0.427\end{array}$ & $\begin{array}{c}0.70(0.43-1.16) \\
P: 0.164\end{array}$ \\
\hline$\geq 24$ & $82 / 199$ & $84 / 219$ & $17 / 65$ & 1.00 & $\begin{array}{l}0.88(0.61-1.27) \\
P: 0.490\end{array}$ & $\begin{array}{l}0.64(0.35-1.17) \\
P: 0.147\end{array}$ & $\begin{array}{c}0.84(0.59-1.19) \\
P: 0.323\end{array}$ & $\begin{array}{l}0.69(0.38-1.23) \\
P: 0.203\end{array}$ \\
\hline
\end{tabular}

${ }^{a}$ The genotyping was successful in 521 (99.81\%) NSCLC cases, and 1030 (99.90\%) controls for MTHFR rs1801133 G>A;

${ }^{\mathrm{b}}$ Adjusted for age, sex, BMI, smoking status and alcohol consumption (besides stratified factors accordingly) in a logistic regression model;

never smoking subgroup: CC vs. TC/TT: adjusted OR $=1.50,95 \%$ CI $1.05-2.14, P=0.025 ; \mathrm{BMI}<24 \mathrm{~kg} / \mathrm{m}^{2}$ group: CC vs. TT: adjusted OR $=1.56,95 \%$ CI $1.01-2.39$, $P=0.044$ and $\mathrm{CC} v$ s. TC/TT: adjusted $\mathrm{OR}=1.56,95 \% \mathrm{CI}$ 1.06-2.29, $P=0.023$; Table 8).

\section{SNP haplotypes}

Using SHEsis software (http://analysis.bio-x.cn) [18], we further constructed seven MTHFR haplotypes (Table 9). Haplotype comparison analysis indicated that MTHFR haplotypes were not associated with the risk of NSCLC.

\section{DISCUSSION}

In the present study, the relationships of MTHFR tagging polymorphisms with the development of NSCLC risk were explored. The results highlighted that MTHFR rs1801133 $\mathrm{G}>\mathrm{A}$ might decrease the risk of overall NSCLC. In addition, we found MTHFR rs1801133 $\mathrm{G}>\mathrm{A}$ variants were associated with the decreased risk of NSCLC in female, $<60$ years and never smoking subgroups. However, we found that MTHFR rs4845882 $\mathrm{G}>\mathrm{A}$ polymorphism was associated with the development of NSCLC in female subgroup. The association between MTHFR rs9651118 T $>$ C polymorphism and the increased the risk of NSCLC was also evident in $<60$ years, never smoking and $\mathrm{BMI}<24 \mathrm{~kg} / \mathrm{m}^{2}$ subgroups.

Variants of MTHFR, which is an important regulator of intracellular folate metabolism, were found that they were associated with the increased level of circulating homocysteine and many diseases involving NSCLC. A number of case-control studies focused on the association of NSCLC with MTHFR SNPs and had controversial findings. Recently, a meta-analysis which included twenty-six studies demonstrated that MTHFR contribute to the risk of NSCLC in Asians and overall populations, but not Caucasians [17]. Another meta-analysis which enrolled 10 studies with 2487 cases and 3228 controls suggested that rs 1801133 $\mathrm{G}>\mathrm{A}$ polymorphism in $M T H F R$ gene may not be a risk factor of NSCLC in Chinese populations; however, the association between this SNP and NSCLC risk might alter in different region of China [16]. Clearly, 
Table 5: Stratified analyses between $M T H F R$ rs3753584 T>C polymorphism and NSCLC risk by sex, age, BMI, smoking status and alcohol consumption

\begin{tabular}{|c|c|c|c|c|c|c|c|c|}
\hline \multirow[t]{2}{*}{ Variable } & \multicolumn{3}{|c|}{$\begin{array}{c}\text { MTHFR rs3753584 T>C (case/ } \\
\text { control) }^{\mathrm{a}}\end{array}$} & \multicolumn{5}{|c|}{ Adjusted $O^{\mathrm{b}}(95 \% \mathrm{CI}) ; P$} \\
\hline & TT & TC & $\mathrm{CC}$ & TT & TC & $\mathrm{CC}$ & TC / CC & CC vs. (TC/TT) \\
\hline \multicolumn{9}{|l|}{ Sex } \\
\hline Male & $231 / 450$ & $52 / 127$ & $4 / 10$ & 1.00 & $\begin{array}{l}0.80(0.54-1.17) \\
P: 0.242\end{array}$ & $\begin{array}{l}0.68(0.20-2.32) \\
P: 0.533\end{array}$ & $\begin{array}{l}0.78(0.54-1.14) \\
P: 0.201\end{array}$ & $\begin{array}{l}0.71(0.21-2.42) \\
P: 0.578\end{array}$ \\
\hline Female & $172 / 350$ & $59 / 89$ & $3 / 3$ & 1.00 & $\begin{array}{l}1.35(0.92-1.97) \\
P: 0.128\end{array}$ & $\begin{array}{l}2.51(0.479-12.75) \\
P: 0.268\end{array}$ & $\begin{array}{l}1.38(0.95-2.01) \\
P: 0.093\end{array}$ & $\begin{array}{l}2.34(0.46-11.88) \\
P: 0.304\end{array}$ \\
\hline \multicolumn{9}{|l|}{ Age } \\
\hline$<60$ & $181 / 362$ & $54 / 110$ & $3 / 3$ & 1.00 & $\begin{array}{l}0.95(0.65-1.40) \\
P: 0.806\end{array}$ & $\begin{array}{l}1.30(0.25-6.67) \\
P: 0.756\end{array}$ & $\begin{array}{l}0.96(0.66-1.41) \\
P: 0.848\end{array}$ & $\begin{array}{l}1.31(0.26-6.73) ; \\
P: 0.745\end{array}$ \\
\hline$\geq 60$ & $222 / 438$ & $57 / 106$ & $4 / 10$ & 1.00 & $\begin{array}{l}1.12(0.77-1.63) \\
P: 0.560\end{array}$ & $\begin{array}{l}0.89(0.26-3.04) \\
P: 0.855\end{array}$ & $\begin{array}{l}1.10(0.76-1.58) \\
P: 0.610\end{array}$ & $\begin{array}{l}0.87(0.26-2.97) \\
P: 0.827\end{array}$ \\
\hline \multicolumn{9}{|c|}{ Smoking status } \\
\hline Never & $240 / 650$ & $73 / 168$ & $4 / 9$ & 1.00 & $\begin{array}{l}1.16(0.84-1.60) \\
P: 0.359\end{array}$ & $\begin{array}{l}1.48(0.44-5.00) \\
P: 0.528\end{array}$ & $\begin{array}{l}1.18(0.86-1.61) \\
P: 0.314\end{array}$ & $\begin{array}{l}1.43(0.43-4.83) \\
P: 0.562\end{array}$ \\
\hline Ever & $163 / 150$ & $38 / 48$ & $3 / 4$ & 1.00 & $\begin{array}{l}0.77(0.47-1.25) \\
P: 0.281\end{array}$ & $\begin{array}{l}0.66(0.14-3.02) \\
P: 0.593\end{array}$ & $\begin{array}{l}0.76(0.47-1.21) \\
P: 0.247\end{array}$ & $\begin{array}{l}0.70(0.15-3.20) \\
P: 0.645\end{array}$ \\
\hline \multicolumn{9}{|l|}{$\begin{array}{l}\text { Alcohol } \\
\text { consumption }\end{array}$} \\
\hline Never & $341 / 735$ & $96 / 202$ & $7 / 11$ & 1.00 & $\begin{array}{l}0.99(0.74-1.32) \\
P: 0.937\end{array}$ & $\begin{array}{l}1.43(0.52-3.92) \\
P: 0.489\end{array}$ & $\begin{array}{l}1.01(0.76-1.33) \\
P: 0.951\end{array}$ & $\begin{array}{l}1.43(0.52-3.92) \\
P: 0.486\end{array}$ \\
\hline Ever & $62 / 65$ & $15 / 14$ & $0 / 2$ & 1.00 & $\begin{array}{l}1.31(0.56-3.07) \\
P: 0.537\end{array}$ & - & $\begin{array}{l}1.08(0.48-2.45) \\
P: 0.852\end{array}$ & - \\
\hline \multicolumn{9}{|l|}{$\operatorname{BMI}\left(\mathrm{kg} / \mathrm{m}^{2}\right)$} \\
\hline$<24$ & $265 / 432$ & $69 / 105$ & $3 / 9$ & 1.00 & $\begin{array}{l}1.04(0.73-1.48) \\
P: 0.830\end{array}$ & $\begin{array}{l}0.47(0.12-1.84) \\
P: 0.278\end{array}$ & $\begin{array}{l}0.99(0.70-1.40) \\
P: 0.960\end{array}$ & $\begin{array}{l}0.47(0.12-1.82) \\
P: 0.272\end{array}$ \\
\hline$\geq 24$ & $138 / 368$ & $42 / 111$ & $4 / 4$ & 1.00 & $\begin{array}{l}0.99(0.65-1.50) \\
P: 0.946\end{array}$ & $\begin{array}{l}3.34(0.80-14.04) \\
P: 0.100\end{array}$ & $\begin{array}{l}1.06(0.70-1.59) \\
P: 0.788\end{array}$ & $\begin{array}{l}3.35(0.80-14.04) \\
P: 0.098\end{array}$ \\
\hline
\end{tabular}

a The genotyping was successful in 521 (100.00\%) NSCLC cases, and 1030 (99.90\%) controls for MTHFR rs3753584 T $>$ C;

${ }^{\mathrm{b}}$ Adjusted for age, sex, BMI, smoking status and alcohol consumption (besides stratified factors accordingly) in a logistic regression model;

these ambiguous findings indicated that the function of MTHFR rs1801133 G>A polymorphism might be varied in different race or even in the different region of the same ethnicity, which suggested large-scale case-control studies in different regions and ethnicities were needed to further explore the potential relationship. It was found that activation and variant frequencies of MTHFR might alter among different region and different latitude with the various ultraviolet-exposure levels [19, 20]. Furthermore, the sample sizes were relatively small in most of included studies. A functional study indicated that MTHFR rs1801133 $\mathrm{G}>\mathrm{A}$ polymorphism was a protective factor of prostate cancer (PC) susceptibility by elevating homocysteine level, promoting cell apoptosis, and inhibiting proliferation of PC cells [21]. In the present study, we found that MTHFR rs1801133 A allele might be a protective factor for NSCLC, which was similar to the findings of previous study conducted in Eastern Chinese Han populations. However, these potential associations were not significant after the Bonferroni correction for multiple comparisons. Thus, our findings should be explained with very cautions.
Rs4845882 G>A, a SNP locate in intron region of $M T H F R$ gene, was strongly complete linkage disequilibrium (LD) with $M T H F R$ rs1801131 A $>$ C polymorphism [( $r 2=$ 0.935); http://gvs.gs.washington.edu/GVS147/]. Wang et al. reported there was no significant correlation between MTHFR rs4845882 G>A polymorphism and gastric cardia carcinoma (GCA) risk [22]. However, another study saw a decreased esophageal squamous cell carcinoma (ESCC) risk in Chinese Han individuals with MTHFR rs4845882 AA genotype [23]. In the present study, we found that MTHFR $4845882 \mathrm{G}>\mathrm{A}$ might be a risk factor for NSCLC in female subgroup. These inconsistent findings may be due to the limited sample size or other confounding factors. In the future, large-scale study with comprehensive functional exploring should be conducted. And the confounding gene or environmental factors also could not be ignored.

In this study, we found MTHFR rs9651118 T>C polymorphism was associated with the NSCLC risk in some subgroups. And recent case-control studies indicated that this SNP might play different roles among different type of cancer. For example, some studies suggested that MTHFR rs9651118 $\mathrm{T}>\mathrm{C}$ polymorphism was associated with the 
Table 6: Stratified analyses between MTHFR rs4845882 G>A polymorphism and NSCLC risk by sex, age, BMI, smoking status and alcohol consumption

\begin{tabular}{|c|c|c|c|c|c|c|c|c|}
\hline \multirow{2}{*}{ Variable } & \multicolumn{3}{|c|}{$\begin{array}{c}\text { MTHFR rs4845882 G>A (case/ } \\
\text { control) }\end{array}$} & \multicolumn{5}{|c|}{ Adjusted $\mathrm{OR}^{\mathrm{b}}(95 \% \mathrm{CI}) ; P$} \\
\hline & GG & GA & $\mathbf{A A}$ & GG & GA & $\mathbf{A A}$ & GA/AA & AA vs. (GA/GG) \\
\hline \multicolumn{9}{|l|}{ Sex } \\
\hline Male & $177 / 349$ & $94 / 212$ & $16 / 26$ & 1.00 & $\begin{array}{l}0.87(0.63-1.21) \\
P: 0.411\end{array}$ & $\begin{array}{l}1.42(0.71-2.85) \\
P: 0.322\end{array}$ & $\begin{array}{l}0.93(0.68-1.26) ; \\
P: 0.627\end{array}$ & $\begin{array}{l}1.49(0.75-2.96) \\
P: 0.254\end{array}$ \\
\hline Female & $132 / 283$ & $97 / 142$ & $5 / 17$ & 1.00 & $\begin{array}{l}1.47(1.05-2.05) \\
\text { P: } 0.025\end{array}$ & $\begin{array}{l}0.68(0.24-1.90) \\
P: 0.460\end{array}$ & $\begin{array}{l}1.39(1.00-1.93) ; \\
P: 0.051\end{array}$ & $\begin{array}{l}0.59(0.21-1.63) \\
P: 0.304\end{array}$ \\
\hline \multicolumn{9}{|l|}{ Age } \\
\hline$<60$ & $136 / 286$ & $92 / 169$ & $10 / 20$ & 1.00 & $\begin{array}{l}1.17(0.83-1.65) \\
P: 0.361\end{array}$ & $\begin{array}{l}1.15(0.50-2.61) \\
P: 0.746\end{array}$ & $\begin{array}{l}1.17(0.84-1.63) ; \\
P: 0.359\end{array}$ & $\begin{array}{l}1.08(0.48-2.42) \\
P: 0.862\end{array}$ \\
\hline$\geq 60$ & $173 / 346$ & $99 / 185$ & $11 / 23$ & 1.00 & $\begin{array}{l}1.07(0.78-1.47) \\
P: 0.685\end{array}$ & $\begin{array}{l}1.12(0.52-2.43) \\
P: 0.777\end{array}$ & $\begin{array}{l}1.07(0.79-1.46) \\
P: 0.653\end{array}$ & $\begin{array}{l}1.09(0.51-2.35) \\
P: 0.820\end{array}$ \\
\hline \multicolumn{9}{|c|}{ Smoking status } \\
\hline Never & $186 / 510$ & $119 / 282$ & $12 / 35$ & 1.00 & $\begin{array}{l}1.16(0.88-1.53) \\
P: 0.301\end{array}$ & $\begin{array}{l}1.09(0.54-2.18) \\
P: 0.815\end{array}$ & $\begin{array}{l}1.15(0.88-1.51) \\
P: 0.313\end{array}$ & $\begin{array}{l}1.03(0.52-2.04) \\
P: 0.940\end{array}$ \\
\hline Ever & $123 / 122$ & $72 / 72$ & $9 / 8$ & 1.00 & $\begin{array}{l}1.02(0.68-1.55) \\
P: 0.914\end{array}$ & $\begin{array}{l}1.21(0.45-3.27) \\
P: 0.705\end{array}$ & $\begin{array}{l}1.04(0.70-1.56) \\
P: 0.842\end{array}$ & $\begin{array}{l}1.20(0.45-3.20) \\
P: 0.714\end{array}$ \\
\hline \multicolumn{9}{|l|}{$\begin{array}{l}\text { Alcohol } \\
\text { consumption }\end{array}$} \\
\hline Never & $260 / 581$ & $168 / 327$ & $16 / 40$ & 1.00 & $\begin{array}{l}1.14(0.89-1.45) \\
P: 0.312\end{array}$ & $\begin{array}{l}0.99(0.53-1.85) \\
P: 0.982\end{array}$ & $\begin{array}{l}1.12(0.88-1.42) \\
P: 0.354\end{array}$ & $\begin{array}{l}0.95(0.51-1.75) \\
P: 0.858\end{array}$ \\
\hline Ever & $49 / 51$ & $23 / 27$ & $5 / 3$ & 1.00 & $\begin{array}{l}0.95(0.47-1.92) \\
P: 0.893\end{array}$ & $\begin{array}{l}2.23(0.47-10.67) \\
P: 0.315\end{array}$ & $\begin{array}{l}1.06(0.54-2.08) ; \\
P: 0.858\end{array}$ & $\begin{array}{l}2.27(0.48-10.64) ; \\
P: 0.298\end{array}$ \\
\hline \multicolumn{9}{|l|}{ BMI $\left(\mathrm{kg} / \mathrm{m}^{2}\right)$} \\
\hline$<24$ & $207 / 338$ & $120 / 190$ & $10 / 18$ & 1.00 & $\begin{array}{l}1.06(0.78-1.42) \\
P: 0.724\end{array}$ & $\begin{array}{l}1.03(0.45-2.35) \\
P: 0.952\end{array}$ & $\begin{array}{l}1.05(0.79-1.41) \\
P: 0.737\end{array}$ & $\begin{array}{l}1.00(0.44-2.28) \\
P: 0.992\end{array}$ \\
\hline$\geq 24$ & $102 / 294$ & $71 / 164$ & $11 / 25$ & 1.00 & $\begin{array}{l}1.24(0.85-1.79) \\
P: 0.262\end{array}$ & $\begin{array}{l}1.26(0.58-2.72) \\
P: 0.557\end{array}$ & $\begin{array}{l}1.24(0.87-1.77) \\
P: 0.236\end{array}$ & $\begin{array}{l}1.16(0.55-2.48) \\
P: 0.697\end{array}$ \\
\hline
\end{tabular}

a The genotyping was successful in 521 (100.00\%) NSCLC cases, and 1030 (99.90\%) controls for MTHFR rs4845882 G>A;

${ }^{\mathrm{b}}$ Adjusted for age, sex, BMI, smoking status and alcohol consumption (besides stratified factors accordingly) in a logistic regression model;

decreased susceptibility of LC and PC [14, 24]. While Tang et al. and Wang et al. observed a null association of MTHFR rs9651118 T $>$ C polymorphism with the risk of ESCC and GCA $[22,23]$. Therefore, whether the T-to-C transition in the intron 2 region does alter the functions of MTHFR gene needs to be further explored.

However, several limitations in our study must be acknowledged. Firstly, other functional SNP loci in the region of the MTHFR gene may be related to NSCLC susceptibility. Unfortunately, because of genotyping cost, we were unable to perform a fine-mapping study focusing on the association between MTHFR SNPS and NSCLC risk. Secondly, the sample size of NSCLC patients was moderate and detailed information of some NSCLC patients was not available. The relationship of MTHFR SNPs with tumor stages or cancer subtypes was not carried out. This could limit the validity of the findings because these potentially factors might not be well understood. Thirdly, selected biases might result in spurious findings because the NSCLC patients and the controls were enrolled from the local hospitals. Finally, other potential gene-environment factors were not considered. Further studies focusing on the interactions of multiple environment and gene factors on NSCLC risk are needed to confirm our findings.

In conclusion, the current study highlights MTHFR rs $1801133 \mathrm{G}>\mathrm{A}$ variants are associated with the decreased risk of NSCLC. However, MTHFR rs4845882 G>A and $M T H F R$ rs9651118 $\mathrm{T}>\mathrm{C}$ polymorphisms may increase the risk of NSCLC. Well-designed large-scale studies are needed to confirm these findings and explore the interactions of gene-gene and gene-environment involved in MTHFR SNPs and NSCLC.

\section{MATERIALS AND METHODS}

\section{Ethics Statement}

This case-control study was conducted in Fujian and Jiangsu Province in Eastern of China. The ethical board approval from Ethics Committee of Fujian Medical University (Fuzhou, China) and Jiangsu University (Zhenjiang, China) was obtained, and all of the participants signed written informed consent. 
Table 7: Stratified analyses between $M T H F R$ rs4846048 A>G polymorphism and NSCLC risk by sex, age, BMI, smoking status and alcohol consumption

\begin{tabular}{|c|c|c|c|c|c|c|c|c|}
\hline \multirow[t]{2}{*}{ Variable } & \multicolumn{3}{|c|}{$\begin{array}{c}\text { MTHFR rs4846048 A }>\mathrm{G} \text { (case/ } \\
\text { control) }\end{array}$} & \multicolumn{5}{|c|}{ Adjusted $\mathrm{OR}^{\mathrm{b}}(95 \% \mathrm{CI}) ; P$} \\
\hline & AA & AG & GG & $\mathbf{A A}$ & AG & GG & AG/GG & GG vs. (AG/AA) \\
\hline \multicolumn{9}{|l|}{ Sex } \\
\hline Male & $233 / 488$ & $51 / 88$ & $3 / 11$ & 1.00 & $\begin{array}{l}1.29(0.86-1.94) ; \\
\quad P: 0.221\end{array}$ & $\begin{array}{c}0.68(0.18-2.64) \\
P: 0.576\end{array}$ & $\begin{array}{l}1.22(0.83-1.81) \\
\quad P: 0.314\end{array}$ & $\begin{array}{l}0.65(0.17-2.52) \\
\quad P: 0.535\end{array}$ \\
\hline Female & $195 / 361$ & $39 / 77$ & $0 / 4$ & 1.00 & $\begin{array}{c}0.97(0.63-1.49) \\
P: 0.884\end{array}$ & - & $\begin{array}{l}0.92(0.60-1.41) \\
P: 0.701\end{array}$ & - \\
\hline \multicolumn{9}{|l|}{ Age } \\
\hline$<60$ & $191 / 393$ & $47 / 74$ & $0 / 8$ & 1.00 & $\begin{array}{c}1.45(0.95-2.22) \\
P: 0.088\end{array}$ & - & $\begin{array}{l}\text { 1.32(0.87-2.00); } \\
\quad P: 0.197\end{array}$ & - \\
\hline$\geq 60$ & $237 / 456$ & $43 / 91$ & $3 / 7$ & 1.00 & $\begin{array}{c}0.88(0.59-1.33) \\
P: 0.555\end{array}$ & $\begin{array}{c}0.94(0.23-3.86) \\
P: 0.936\end{array}$ & $\begin{array}{c}0.89(0.60-1.32) \\
P: 0.558\end{array}$ & $\begin{array}{c}0.96(0.24-3.93) \\
P: 0.959\end{array}$ \\
\hline \multicolumn{9}{|c|}{ Smoking status } \\
\hline Never & $262 / 676$ & $53 / 140$ & $2 / 11$ & 1.00 & $\begin{array}{c}0.98(0.69-1.40) ; \\
\quad P: 0.925\end{array}$ & $\begin{array}{c}0.60(0.13-2.79) \\
P: 0.512\end{array}$ & $\begin{array}{l}0.96(0.68-1.36) \\
\quad P: 0.810\end{array}$ & $\begin{array}{c}0.60(0.13-2.79) \\
P: 0.513\end{array}$ \\
\hline Ever & $166 / 173$ & $37 / 25$ & $1 / 4$ & 1.00 & $\begin{array}{c}1.54(0.89-2.68) ; \\
P: 0.126\end{array}$ & $\begin{array}{c}0.31(0.03-2.87) \\
P: 0.302\end{array}$ & $\begin{array}{l}1.39(0.81-2.36) \\
P: 0.230\end{array}$ & $\begin{array}{c}0.29(0.03-2.67) \\
P: 0.274\end{array}$ \\
\hline \multicolumn{9}{|l|}{$\begin{array}{l}\text { Alcohol } \\
\text { consumption }\end{array}$} \\
\hline Never & $366 / 779$ & $76 / 156$ & $2 / 13$ & 1.00 & $\begin{array}{c}1.06(0.77-1.45) ; \\
P: 0.723\end{array}$ & $\begin{array}{c}0.41(0.09-1.89) ; \\
P: 0.254\end{array}$ & $\begin{array}{c}1.01(0.75-1.38) \\
\quad P: 0.931\end{array}$ & $\begin{array}{c}0.41(0.09-1.87) \\
P: 0.248\end{array}$ \\
\hline Ever & $62 / 70$ & $14 / 9$ & $1 / 2$ & 1.00 & $\begin{array}{c}1.95(0.77-4.96) ; \\
P: 0.162\end{array}$ & $\begin{array}{c}0.57(0.05-7.23) \\
P: 0.664\end{array}$ & $\begin{array}{c}\text { 1.70(0.71-4.09); } \\
\quad P: 0.238\end{array}$ & $\begin{array}{l}0.52(0.04-6.52) \\
P: 0.610\end{array}$ \\
\hline \multicolumn{9}{|l|}{$\operatorname{BMI}\left(\mathrm{kg} / \mathrm{m}^{2}\right)$} \\
\hline$<24$ & $281 / 455$ & $55 / 86$ & $1 / 5$ & 1.00 & $\begin{array}{c}1.10(0.75-1.62) \\
P: 0.629\end{array}$ & $\begin{array}{l}0.55(0.06-4.92) \\
P: 0.591\end{array}$ & $\begin{array}{c}1.08(0.74-1.57) \\
\quad P: 0.708\end{array}$ & $\begin{array}{l}0.54(0.06-4.83) \\
P: 0.579\end{array}$ \\
\hline$\geq 24$ & $147 / 394$ & $35 / 79$ & $2 / 10$ & 1.00 & $\begin{array}{c}1.20(0.76-1.89) ; \\
P: 0.433\end{array}$ & $\begin{array}{l}0.41(0.08-2.02) \\
P: 0.274\end{array}$ & $\begin{array}{c}1.10(0.71-1.71) \\
P: 0.678\end{array}$ & $\begin{array}{c}0.40(0.08-1.96) \\
P: 0.258\end{array}$ \\
\hline
\end{tabular}

${ }^{a}$ The genotyping was successful in $521(100.00 \%)$ NSCLC cases, and $1030(99.90 \%)$ controls for MTHFR rs $4846048 \mathrm{~A}>\mathrm{G}$; ${ }^{\mathrm{b}}$ Adjusted for age, sex, BMI, smoking status and alcohol consumption (besides stratified factors accordingly) in a logistic regression model.

\section{Subjects}

All sporadic NSCLC patients were enrolled from the Affiliated Union Hospital of Fujian Medical University and the Affiliated People's Hospital of Jiangsu University. Our study consisted of 521 NSCLC patients (mean age $59.76 \pm 10.71$ years) from January 2014 to December 2016. The diagnosis was confirmed based on pathological findings. For comparison, 1,030 non-cancer controls (mean age $60.34 \pm 9.11$ years) were recruited from normal volunteers who conducted health check in the Physical Examination Center of these hospitals. The controls had no history of autoimmune disorders or personal malignancy, and were frequency well-matched to patients by age and sex. The included risk factors (tobacco consumption and drinking) and demographic details of the NSCLC patients and controls were obtained by using a structured questionnaire. The data are listed in Table 1.

\section{Preparation of genomic DNA}

Lymphocytes were separated from EDTAanticoagulated whole blood. Genomic DNA was carefully extracted using the Promega DNA kit (Promega, Madison, USA).

\section{SNP selection}

SNPs were selected using Haploview 4.2 software and the HapMap database. Five haplotype-tagging SNPs of MTHFR gene (rs3753584 T>C, rs4845882 G>A, rs $1801133 \mathrm{G}>\mathrm{A}$, rs4846048 $\mathrm{A}>\mathrm{G}$ and rs9651118 $\mathrm{T}>\mathrm{C}$ ) were selected, with MAF $>5 \%$, call rate $\geq 95 \%$, HWE $P \geq 0.05$ and pair-wise $\mathrm{r}^{2}<0.8$ for each SNP pair. In total, the five tagging SNPs were selected by spaning the entire MTHFR gene region (upstream and downstream extending $5 \mathrm{~Kb}$, respectively). The primary information of the selected SNPs is presented in Table 2. 
Table 8: Stratified analyses between $M T H F R$ rs9651118 T $>$ C polymorphism and NSCLC risk by sex, age, BMI, smoking status and alcohol consumption

\begin{tabular}{|c|c|c|c|c|c|c|c|c|}
\hline \multirow[t]{2}{*}{ Variable } & \multicolumn{3}{|c|}{$\begin{array}{c}\text { MTHFR rs9651118 } \mathrm{T}>\mathrm{C} \\
\text { (case/control) }^{\mathrm{a}}\end{array}$} & \multicolumn{5}{|c|}{ Adjusted OR $(95 \%$ CI $) ; P$} \\
\hline & TT & $\mathbf{T C}$ & $\mathrm{CC}$ & TT & TC & $\mathrm{CC}$ & TC / CC & CC vs. (TC/TT) \\
\hline \multicolumn{9}{|l|}{ Sex } \\
\hline Male & $110 / 209$ & $133 / 300$ & $44 / 78$ & 1.00 & $\begin{array}{l}0.85(0.61-1.18) \\
P: 0.330\end{array}$ & $\begin{array}{l}1.11(0.69-1.76) ; \\
P: 0.676\end{array}$ & $\begin{array}{l}0.90(0.66-1.23) \\
P: 0.503\end{array}$ & $\begin{array}{l}1.21(0.79-1.86) ; \\
P: 0.381\end{array}$ \\
\hline Female & $77 / 169$ & $112 / 213$ & $45 / 60$ & 1.00 & $\begin{array}{l}1.05(0.73-1.51) \\
P: 0.794\end{array}$ & $\begin{array}{l}1.48(0.92-2.39) \\
P: 0.109\end{array}$ & $\begin{array}{l}1.15(0.81-1.61) \\
P: 0.435\end{array}$ & $\begin{array}{l}1.44(0.94-2.22) \text {; } \\
P: 0.098\end{array}$ \\
\hline \multicolumn{9}{|l|}{ Age } \\
\hline$<60$ & $82 / 163$ & $110 / 255$ & $46 / 57$ & 1.00 & $\begin{array}{l}0.90(0.62-1.29) \\
P: 0.549\end{array}$ & $\begin{array}{l}1.64(1.00-2.69) \\
\text { P: } 0.049\end{array}$ & $\begin{array}{l}1.03(0.73-1.45) \\
P: 0.868\end{array}$ & $\begin{array}{l}1.75(1.12-2.74) ; \\
P: 0.014\end{array}$ \\
\hline$\geq 60$ & $105 / 215$ & $135 / 258$ & $43 / 81$ & 1.00 & $\begin{array}{l}1.00(0.72-1.39) \\
P: 1.00\end{array}$ & $\begin{array}{l}1.05(0.66-1.65) \\
P: 0.848\end{array}$ & $\begin{array}{l}1.01(0.74-1.38) \\
P: 0.946\end{array}$ & $\begin{array}{l}1.05(0.69-1.59) \\
P: 0.834\end{array}$ \\
\hline \multicolumn{9}{|l|}{$\begin{array}{l}\text { Smoking } \\
\text { status }\end{array}$} \\
\hline Never & $111 / 305$ & $146 / 412$ & $60 / 110$ & 1.00 & $\begin{array}{l}0.93(0.69-1.25) \\
P: 0.646\end{array}$ & $\begin{array}{l}1.45(0.98-2.14) \\
P: 0.066\end{array}$ & $\begin{array}{l}1.04(0.79-1.37) \\
P: 0.791\end{array}$ & $\begin{array}{l}1.50(1.05-2.14) \text {; } \\
P: 0.025\end{array}$ \\
\hline Ever & $76 / 73$ & 99/101 & $29 / 28$ & 1.00 & $\begin{array}{l}0.95(0.62-1.46) \\
P: 0.819\end{array}$ & $\begin{array}{l}0.97(0.52-1.80) \\
P: 0.918\end{array}$ & $\begin{array}{l}0.96(0.64-1.44) \\
P: 0.824\end{array}$ & $\begin{array}{l}1.00(0.56-1.76) ; \\
P: 0.989\end{array}$ \\
\hline \multicolumn{9}{|l|}{$\begin{array}{l}\text { Alcohol } \\
\text { consumption }\end{array}$} \\
\hline Never & $159 / 340$ & $205 / 479$ & $80 / 129$ & 1.00 & $\begin{array}{l}0.87(0.67-1.13) \\
P: 0.296\end{array}$ & $\begin{array}{l}1.25(0.88-1.78) \\
P: 0.212\end{array}$ & $\begin{array}{l}0.95(0.74-1.21) \\
P: 0.677\end{array}$ & $\begin{array}{l}1.35(0.99-1.86) ; \\
P: 0.062\end{array}$ \\
\hline Ever & $28 / 38$ & $40 / 34$ & $9 / 9$ & 1.00 & $\begin{array}{l}1.66(0.83-3.30) \\
P: 0.153\end{array}$ & $\begin{array}{l}1.45(0.49-4.24) \\
P: 0.501\end{array}$ & $\begin{array}{l}1.61(0.83-3.11) \\
P: 0.155\end{array}$ & $\begin{array}{l}1.11(0.40-3.03) \text {; } \\
P: 0.845\end{array}$ \\
\hline \multicolumn{9}{|l|}{ BMI $\left(\mathrm{kg} / \mathrm{m}^{2}\right)$} \\
\hline$<24$ & $108 / 187$ & $165 / 287$ & $64 / 72$ & 1.00 & $\begin{array}{l}0.99(0.72-1.36) \\
P: 0.967\end{array}$ & $\begin{array}{l}1.56(1.01-2.39) \\
\text { P: } 0.044\end{array}$ & $\begin{array}{l}1.10(0.81-1.49) \\
P: 0.532\end{array}$ & $\begin{array}{l}1.56(1.06-2.29) \\
P: 0.023\end{array}$ \\
\hline$\geq 24$ & $79 / 191$ & $80 / 226$ & $25 / 66$ & 1.00 & $\begin{array}{l}0.85(0.58-1.25) \\
P: 0.406\end{array}$ & $\begin{array}{l}0.95(0.55-1.64) \\
P: 0.850\end{array}$ & $\begin{array}{l}0.87(0.61-1.25) \\
P: 0.456\end{array}$ & $\begin{array}{l}1.03(0.62-1.72) \\
P: 0.908\end{array}$ \\
\hline
\end{tabular}

${ }^{a}$ The genotyping was successful in 521 (100.00\%) NSCLC cases, and 1030 (99.90\%) controls for MTHFR rs9651118 T $>$ C;

${ }^{\mathrm{b}}$ Adjusted for age, sex, BMI, smoking status and alcohol consumption (besides stratified factors accordingly) in a logistic regression model;

\section{Genotyping}

All SNPs were genotyped using the SNPscan ${ }^{\mathrm{TM}}$ genotyping assay (Genesky Biotechologies Inc., Shanghai, China), which is a double ligation and multiplex fluorescence PCR [25]. The accuracy of genotyping results were verified by reanalyzing the genotypes in $4 \%$ random samples.

\section{Statistical analysis}

Age of NSCLC patients and controls was described as the mean \pm deviation (SD). And a Student's $t$-test was used to examine the difference for age. The deviation from HWE was assessed using an online goodness-offit chi-squared test (http://ihg.gsf.de/cgi-bin/hw/hwa1. pl) in controls [26-32]. Using different genetic models (additive, homozygote, dominant, recessive), the genotype frequencies of the subjects were compared using the chisquared $\left(\chi^{2}\right)$ test. Multivariate logistic regression analysis was harnessed to assess the risk of mutant genotype with respect to wild type and considered established confounders such as age, sex, smoking, BMI and drinking status. Crude/adjusted ORs with their 95\% CIs were used to assess the difference for the genotype distribution. All data were analyzed using SAS software (Version 9.4; SAS Institute Inc., Cary, NC, USA). SHESIS program (Bio-X Inc., Shanghai, China, http://analysis.bio-X.cn/ myAnalysis.php)] [18] was used to construct haplotypes of MTHFR gene. The association of MTHFR haplotypes with NSCLC risk was estimated as crude ORs with the corresponding $95 \%$ CIs. In this study, the threshold for significance was $P<0.05$ (two tailed). We used Bonferroni correction to perform multiple comparisons [33]. 
Table 9: MTHFR haplotype frequencies (\%) in cases and controls and risk of NSCLC

\begin{tabular}{|c|c|c|c|c|c|c|}
\hline & \multicolumn{2}{|c|}{ Case $(n=1042)$} & \multicolumn{2}{|c|}{ Control $(n=2060)$} & \multirow{2}{*}{ Crude OR (95\% CI) } & \multirow{2}{*}{$P$} \\
\hline & $n$ & $\%$ & $n$ & $\%$ & & \\
\hline G T G A C & 414 & 39.81 & 774 & 37.63 & 1.00 & \\
\hline A T G A T & 317 & 30.48 & 687 & 33.40 & $0.86(0.72-1.03)$ & 0.105 \\
\hline G CAAT & 121 & 11.63 & 220 & 10.70 & $1.03(0.80-1.32)$ & 0.828 \\
\hline G T A G T & 92 & 8.85 & 185 & 8.99 & $0.93(0.70-1.23)$ & 0.606 \\
\hline G T G A T & 68 & 6.54 & 131 & 6.37 & $0.97(0.71-1.33)$ & 0.853 \\
\hline G TAA T & 13 & 1.25 & 21 & 1.02 & $1.16(0.57-2.34)$ & 0.683 \\
\hline Others & 15 & 1.44 & 39 & 1.90 & $0.72(0.39-1.32)$ & 0.285 \\
\hline
\end{tabular}

With the order of rs $1801133 \mathrm{G}>\mathrm{A}, \mathrm{rs} 3753584 \mathrm{~T}>\mathrm{C}$, rs $4845882 \mathrm{G}>\mathrm{A}, \mathrm{rs} 4846048 \mathrm{~A}>\mathrm{G}$ and rs9651118 $\mathrm{T}>\mathrm{C}$ in gene position.

\section{ACKNOWLEDGMENTS}

We appreciate all subjects who participated in this study. We wish to thank Dr. Yan Liu (Genesky Biotechnologies Inc., Shanghai, China) for technical support.

\section{CONFLICTS OF INTEREST}

The authors have no potential financial conflicts of interest.

\section{GRANT SUPPORT}

This study was supported by Natural Science Foundation of Universities and Colleges of Jiangsu Province (Grant No. 16KJB310002), Young and Middle-aged Talent Training Project of Health Development Planning Commission in Fujian Province (2016-ZQN-25 and 2014-ZQN-JC-11), Medical Innovation Project of Fujian Province (2014-CX-15 and 2014-CX-18), Nursery Garden Project of Fujian Medical University (2015MP020) and Science and Technology Project of Fujian Province (2060203).

\section{REFERENCES}

1. Hamra GB, Guha N, Cohen A, Laden F, RaaschouNielsen O, Samet JM, Vineis P, Forastiere F, Saldiva P, Yorifuji T, Loomis D. Outdoor particulate matter exposure and lung cancer: a systematic review and metaanalysis. Environ Health Perspect. 2014; 122:906-11. https://doi.org/10.1289/ehp.1408092.

2. Kopp M, Morisset R, Rychlik M. Characterization and Interrelations of One-Carbon Metabolites in Tissues, Erythrocytes, and Plasma in Mice with Dietary Induced Folate Deficiency. Nutrients. 2017; 9. https://doi. org/10.3390/nu9050462.

3. Bird AP. The relationship of DNA methylation to cancer. Cancer Surv. 1996; 28:87-101.

4. Zingg JM, Jones PA. Genetic and epigenetic aspects of DNA methylation on genome expression, evolution, mutation and carcinogenesis. Carcinogenesis. 1997; 18:869-82.
5. Baylin SB. Tying it all together: epigenetics, genetics, cell cycle, and cancer. Science. 1997; 277:1948-9.

6. Igari S, Ohtaki A, Yamanaka Y, Sato Y, Yohda M, Odaka M, Noguchi K, Yamada K. Properties and crystal structure of methylenetetrahydrofolate reductase from Thermus thermophilus HB8. PLoS One. 2011; 6:e23716. https://doi. org/10.1371/journal.pone.0023716.

7. Marini NJ, Gin J, Ziegle J, Keho KH, Ginzinger D, Gilbert DA, Rine J. The prevalence of folate-remedial MTHFR enzyme variants in humans. Proc Natl Acad Sci U S A. 2008; 105:8055-60. https://doi.org/10.1073/pnas.0802813105.

8. Rai V. Folate pathway gene MTHFR C677T polymorphism and risk of lung cancer in Asian populations. Asian Pac J Cancer Prev. 2014; 15:9259-64.

9. Cheng Z, Wang W, Dai LL, Kang Y. MTHFR C667T polymorphism association with lung cancer risk in Henan province: a case-control study. Asian Pac J Cancer Prev. 2012; 13:2491-4.

10. Cui LH, Yu Z, Zhang TT, Shin MH, Kim HN, Choi JS. Influence of polymorphisms in MTHFR 677 C-$>$ T, TYMS 3R-->2R and MTR 2756 A-->G on NSCLC risk and response to platinum-based chemotherapy in advanced NSCLC. Pharmacogenomics. 2011; 12:797808. https://doi.org/10.2217/pgs.11.27.

11. Al-Motassem Y, Shomaf M, Said I, Berger S, Ababneh N, Diab O, Obeidat N, Awidi A. Allele and Genotype Frequencies of the Polymorphic Methylenetetrahydrofolate Reductase and Lung Cancer in ther Jordanian Population: a Case Control Study. Asian Pac J Cancer Prev. 2015; 16:3101-9.

12. Cavic M, Krivokuca A, Spasic J, Brotto K, Malisic E, Radosavljevic D, Radulovic S, Jankovic R. The influence of methylenetetrahydrofolate reductase and thymidylate synthetase gene polymorphisms on lung adenocarcinoma occurrence. J BUON. 2014; 19:1024-8.

13. Yilmaz M, Kacan T, Sari I, Kilickap S. Lack of association between the MTHFRC677T polymorphism and lung cancer in a Turkish population. Asian Pac J Cancer Prev. 2014; 15:6333-7.

14. Swartz MD, Peterson CB, Lupo PJ, Wu X, Forman MR, Spitz MR, Hernandez LM, Vannucci M, Shete S. 
Investigating multiple candidate genes and nutrients in the folate metabolism pathway to detect genetic and nutritional risk factors for lung cancer. PLoS One. 2013; 8:e53475. https://doi.org/10.1371/journal.pone.0053475.

15. Liu ZB, Wang LP, Shu J, Jin C, Lou ZX. Methylenetetrahydrofolate reductase 677TT genotype might be associated with an increased lung cancer risk in Asians. Gene. 2013; 515:214-9. https://doi. org/10.1016/j.gene.2012.11.036.

16. Wang $\mathrm{X}$, Yue $\mathrm{K}$, Hao L. Meta-analysis of methylenetetrahydrofolate reductase polymorphism and lung cancer risk in Chinese. Int J Clin Exp Med. 2015; 8:1521-5.

17. Yang Y, Yang LJ, Deng MZ, Luo YY, Wu S, Xiong L, Wang D, Liu Y, Liu H. MTHFR C677T and A1298C polymorphisms and risk of lung cancer: a comprehensive evaluation. Genet Mol Res. 2016; 15. https://doi. org/10.4238/gmr.15027615.

18. Shi YY, He L. SHEsis, a powerful software platform for analyses of linkage disequilibrium, haplotype construction, and genetic association at polymorphism loci. Cell Res. 2005; 15:97-8. https://doi.org/10.1038/ sj.cr.7290272.

19. Botto LD, Yang Q. 5,10-Methylenetetrahydrofolate reductase gene variants and congenital anomalies: a HuGE review. Am J Epidemiol. 2000; 151:862-77.

20. Jablonski NG, Chaplin G. Colloquium paper: human skin pigmentation as an adaptation to UV radiation. Proc Natl Acad Sci U S A. 2010; 10:8962-8. https://doi. org/10.1073/pnas.0914628107.

21. Wu JL, Zhou SX, Zhao R, Zhang X, Chang K, Gu CY, Gan HL, Dai B, Zhu Y, Zhang HL, Shi GH, Qu YY, Zhao JY, et al. MTHFR c.677C $>$ T Inhibits Cell Proliferation and Decreases Prostate Cancer Susceptibility in the Han Chinese Population in Shanghai. Sci Rep. 2016; 6:36290. https://doi.org/10.1038/srep36290.

22. Wang Y, Chen S, Kang M, Tang W, Gu H, Yin J, Huang Z. Genetic variations in MTHFR and gastric cardia adenocarcinoma susceptibility in the Chinese Han population. Int J Clin Exp Med. 2015; 8:18936-44.

23. Tang W, Zhang S, Qiu H, Wang L, Sun B, Yin J, Gu H. Genetic variations in MTHFR and esophageal squamous cell carcinoma susceptibility in Chinese Han population. Med Oncol. 2014; 31:915. https://doi.org/10.1007/ s12032-014-0915-6.

24. Lin VC, Lu TL, Yin HL, Yang SF, Lee YC, Liu CC, Huang CY, Yu CC, Chang TY, Huang SP, Bao BY. Prognostic
Relevance of Methylenetetrahydrofolate Reductase Polymorphisms for Prostate Cancer. Int J Mol Sci. 2016; 17. https://doi.org/10.3390/ijms17121996.

25. Yin J, Wang X, Wei J, Wang L, Shi Y, Zheng L, Tang W, Ding G, Liu C, Liu R, Chen S, Xu Z, Gu H. Interleukin 12B rs3212227 $\mathrm{T}>\mathrm{G}$ polymorphism was associated with an increased risk of gastric cardiac adenocarcinoma in a Chinese population. Dis Esophagus. 2015; 28:291-8. https://doi.org/10.1111/dote.12189.

26. Zhang S, Wang Y, Jiang H, Liu C, Gu H, Chen S, Kang M, Tang W. Association between the CD28 IVS3 + 17T $>C$ (rs3116496) polymorphism and cancer susceptibility: a meta-analysis involving 8,843 subjects. Int J Clin Exp Med. 2015; 8:17353-61.

27. Zhang S, Wang Y, Jiang H, Liu C, Sun B, Chen S, Kang M, Tang W. Peroxisome proliferator-activated receptor gamma rs $1801282 \mathrm{C}>\mathrm{G}$ polymorphism is associated with polycystic ovary syndrome susceptibility: a meta-analysis involving 7,069 subjects. Int J Clin Exp Med. 2015; 8:17418-29.

28. Tang W, Wang Y, Jiang H, Liu P, Liu C, Gu H, Chen S, Kang M. Programmed death-1 (PD-1) rs2227981 C > T polymorphism is associated with cancer susceptibility: a meta-analysis. Int J Clin Exp Med. 2015; 8:22278-85.

29. Qiu H, Cheng C, Wang Y, Kang M, Tang W, Chen S, $\mathrm{Gu} \mathrm{H}$, Liu C, Chen Y. Investigation of cyclin D1 rs9344 $\mathrm{G}>\mathrm{A}$ polymorphism in colorectal cancer: a meta-analysis involving 13,642 subjects. Onco Targets Ther. 2016; 9:6641-50. https://doi.org/10.2147/OTT.S116258.

30. Tang W, Qiu H, Ding H, Sun B, Wang L, Yin J, Gu H. Association between the STK15 F31I polymorphism and cancer susceptibility: a meta-analysis involving 43,626 subjects. PLoS One. 2013; 8:e82790. https://doi. org/10.1371/journal.pone.0082790.

31. Tang W, Qiu H, Jiang H, Sun B, Wang L, Yin J, Gu H. Lack of association between cytotoxic T-lymphocyte antigen 4 (CTLA-4) -1722T/C (rs733618) polymorphism and cancer risk: from a case-control study to a meta-analysis. PLoS One. 2014; 9:e94039. https://doi.org/10.1371/journal. pone. 0094039 .

32. Tang W, Wang $\mathrm{Y}$, Chen $\mathrm{S}$, Lin J, Chen B, Yu S, Chen $\mathrm{Y}, \mathrm{Gu} \mathrm{H}$, Kang $\mathrm{M}$. Investigation of Cytotoxic T-lymphocyte antigen 4 Polymorphisms in Gastric Cardia Adenocarcinoma. Scand J Immunol. 2016; 83:212-8. https://doi.org/10.1111/sji.12409.

33. Bland JM, Altman DG. Multiple significance tests: the Bonferroni method. BMJ. 1995; 310:170. 